\title{
Crohn's disease and smoldering multiple myeloma: a case report and literature review
}

\author{
So Young Park ${ }^{1}$, Jae Min Kim ${ }^{1}$, Hyun Joon $\mathrm{Kang}^{1}$, Minje $\mathrm{Kim}^{2}$, Jae Joon $\mathrm{Han}^{3}$, Chi Hoon Maeng ${ }^{3}$, \\ Sun Kyung Baek ${ }^{3}$, Hwi-Joong Yoon ${ }^{3}$, Si-Young Kim ${ }^{3}$, Hyo Jong Kim ${ }^{4}$ \\ ${ }^{1}$ Department of Internal Medicine, Kyung Hee University School of Medicine, Seoul, ${ }^{2}$ Department of Internal Medicine, Graduate School, \\ Kyung Hee University, Seoul, Divisions of ${ }^{3}$ Medical Oncology and Hematology and ${ }^{4}$ Gastroenterology, Department of Internal Medicine, \\ Kyung Hee University School of Medicine, Seoul, Korea
}

Crohn's disease (CD) is a chronic inflammatory bowel disease (IBD) that presents with abdominal pain, weight loss, and diarrhea. Although the etiology has not been fully elucidated, both environmental and genetic causes are known to be involved. In chronic inflammatory conditions such as IBD, B lymphocytes are chronically stimulated, and they induce monoclonal expansion of plasma cells, sometimes resulting in monoclonal gammopathy of undetermined significance. Immunomodulators that are commonly used to control inflammation, such as tumor necrosis factor- $\alpha$ (TNF- $\alpha$ ) blockers could increase the possibility of hematologic malignancy. The pathogenesis of multiple myeloma in association with TNF- $\alpha$ inhibitor therapy is attributed to decreased apoptosis of plasma cell populations. Here, we describe a case of a 36-year-old male patient who was diagnosed with immunoglobulin A subtype smoldering multiple myeloma during the treatment for CD with infliximab and adalimumab. We report this case along with a review of the literature on cases of multiple myeloma that occurred in conjunction with CD.

(Intest Res 2017;15:249-254)

Key Words: Crohn disease; Multiple myeloma; Tumor necrosis factor-alpha

\section{INTRODUCTION}

Crohn's disease (CD) is a type of chronic intractable IBD caused by chronic immune dysregulation. ${ }^{1}$ In chronic inflammatory conditions such as IBD, B lymphocytes are chronically stimulated, and they induce monoclonal expansion of plasma cells, sometimes resulting in monoclonal gammopathy of undetermined significance (MGUS). ${ }^{2}$ Since many cases of multiple myeloma (MM) arise from MGUS as a consequence of several genetic alterations, it is possible that a causal association exists between IBD and $\mathrm{MM}^{3}{ }^{3}$

Received August 24, 2015. Revised October 22, 2015.

Accepted October 22, 2015. Published online March 31, 2017

Correspondence to Chi Hoon Maeng, Division of Medical Oncology and

Hematology, Department of Internal Medicine, Kyung Hee University

Hospital, Kyung Hee University School of Medicine, 23 Kyungheedae-ro,

Dongdaemun-gu, Seoul 02447, Korea. Tel: +82-2-958-2965, Fax: +82-2-

968-1848,E-mail:mchihoon@khu.ac.kr

Financial support: None. Conflict of interest: None.
The dramatic effectiveness of tumor necrosis factor- $\alpha$ (TNF- $\alpha$ ) inhibitors in IBD has contributed to their popular use. Unfortunately, several reports have suggested increased risk of malignancy during TNF- $\alpha$ inhibitor therapy, and the development of MM is not an exception. The pathogenesis of MM in association with TNF- $\alpha$ inhibitor therapy is attributed to decreased apoptosis of plasma cell populations. ${ }^{4-6}$ Here, we describe a case of smoldering multiple myeloma (SMM) diagnosed when a young male patient was receiving TNF- $\alpha$ blocker therapy for $\mathrm{CD}$. We also provide a concise literature review about MM development in patients with $\mathrm{CD}^{1,7-12}$

\section{CASE REPORT}

A 36-year-old male patient was referred for pancytopenia. He had CD since 1998 presenting with diarrhea and anal fistula. The Montreal classification system is widely used for IBD and uses three parameters: age at onset, anatomical

\footnotetext{
๑ Copyright 2017. Korean Association for the Study of Intestinal Diseases. All rights reserved.

This is an Open Access article distributed under the terms of the Creative Commons Attribution Non-Commercial License (http://creativecommons.org/licenses/by-nc/4.0)

which permits unrestricted non-commercial use, distribution, and reproduction in any medium, provided the original work is properly cited.
} 
location, and disease behavior. In addition, a scoring system called CDAI is used to determine the severity of disease activity. At the time of diagnosis, the disease status was classified as A2-L3-B3p according to the Montreal classification, but the initial CDAI was not mentioned in the medical records. After two anal fistulectomies, he was simultaneously treated with azathioprine, mesalazine, and corticosteroid. However, remission was not achieved despite the combination treatment. Therefore, $300 \mathrm{mg}$ infliximab was administered at 0,2 , and 6 weeks, followed by 300 mg every 8 weeks since 2010. However, despite administration of infliximab, the CDAI score remained at 220 or higher, and the patient showed aspects of progressive colonic stricture.

In August 2012, he was transferred to the Division of Gastroenterology at Kyung Hee University Hospital. Mesalazine, azathioprine, and a double dose of infliximab (600 mg) were used for treatment; however, even with this aggressive treatment, the patient was not able to achieve remission. Given the intractability of CD, the patient was treated with ustekinumab, a monoclonal antibody targeting interleukin-12 and -23, in the context of a clinical trial. Despite serial ustekinumab administration, there was no response to treatment. Therefore, we performed ileocecectomy and strictureplasty in May 2013, followed by concomitant treatment with azathioprine (50 mg per day), mesalazine (4 g per day), and adalimumab (160 mg per day initially, followed by 80 mg 2 weeks later, followed by $40 \mathrm{mg}$ every other week) for 9 months.

On follow-up observation, onset of pancytopenia was detected in February 2014. Administration of adalimumab, azathioprine, and mesalazine was stopped for 3 months. Despite discontinuing treatment with drugs that cause my- elosuppression for a considerable period, pancytopenia continued, and he was referred to the department of hematooncology for examination of persistent pancytopenia.

Complete blood cell count results showed hemoglobin level of $5.6 \mathrm{~g} / \mathrm{dL}$, white blood cell count of $2.55 \times 10^{9} / \mathrm{L}$, and platelet count of $82 \times 10^{9} / \mathrm{L}$. Blood chemistry results were as follows: total protein level, $6.8 \mathrm{~g} / \mathrm{dL}$; serum albumin level, $3.7 \mathrm{~g} / \mathrm{dL}$; CRP level, less than $0.3 \mathrm{mg} / \mathrm{dL}$; and electrolyte and renal function within normal limits. Serum IgA level of the patient increased to $9.7 \mathrm{~g} / \mathrm{L}$ (reference value, $0.7-4.0 \mathrm{~g} / \mathrm{L}$ ), and IgG and IgM levels were $9.5 \mathrm{~g} / \mathrm{L}$ (reference value, $7-16$ $\mathrm{g} / \mathrm{L}$ ) and $0.78 \mathrm{~g} / \mathrm{L}$ (reference value, $0.4-2.3 \mathrm{~g} / \mathrm{L}$ ), respectively, which were within the range of reference values. Serum protein electrophoresis revealed an M-protein peak at $0.48 \mathrm{~g} /$ dL (Fig. 1A). Immunofixation showed monoclonal IgA and $\kappa$ bands (Fig. 1B). Serum free light chain concentrations included a free $\kappa$ concentration of $31.8 \mathrm{mg} / \mathrm{L}$ and a free $\lambda$ concentration of $32.1 \mathrm{mg} / \mathrm{L}$ with a serum free $\kappa / \lambda$ ratio of 0.991 . Bone marrow study revealed that the proportion of plasma cells was $14.0 \%$ (Fig. 2). Chromosomal study showed no cytogenetic abnormalities.

In this patient, anemia could be attributed to organ damage due to MM; however, considering that he had $\mathrm{CD}$, the anemia could also be attributed to chronic gastrointestinal bleeding. Therefore, additional studies including iron profile were performed to evaluate his condition. The serum iron level was $21 \mu \mathrm{g} / \mathrm{dL}$, total iron-binding capacity was $468 \mu \mathrm{g} /$ $\mathrm{dL}$, ferritin level was $1.6 \mathrm{ng} / \mathrm{mL}$, and transferrin saturation was at $4.5 \%$. Thus, we concluded that he had a concomitant iron deficiency anemia. The patient received iron intravenously and orally for 6 months, and the hemoglobin level increased to $14 \mathrm{~g} / \mathrm{dL}$.
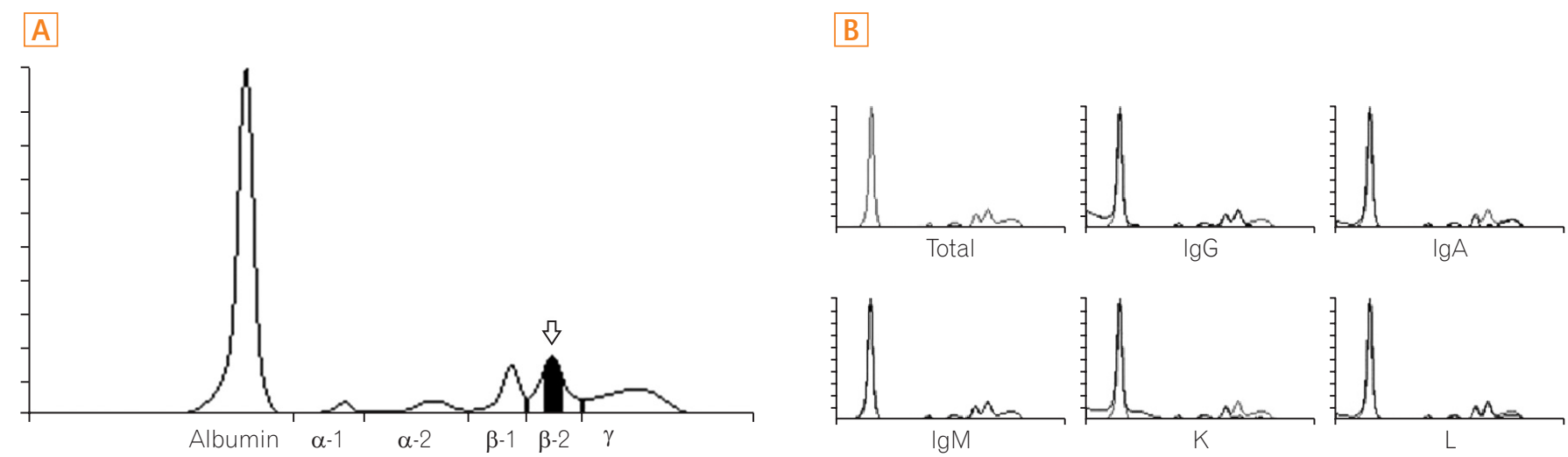

Fig. 1. Serum protein electrophoresis and immunofixation. (A) Serum protein electrophoresis. It shows abnormal zone of restriction in $\beta$-2 region. The $\beta-2$ globulin region represented $13.1 \%$ of total proteins $(7.40 \mathrm{~g} / \mathrm{dL})$. This finding is suggestive of monoclonal gammopathy. The amount of $\mathrm{M}$-peak is estimated to b $0.48 \mathrm{~g} / \mathrm{dL}$ (white arrow). (B) Serum immunofixation electrophoresis. Serum immunotyping shows abnormal zone of restriction in lgA and $\kappa$ type. 
SMM is differentiated from MM based on the lack of endorgan damage. It requires close follow-up, but does not require treatment. SMM is defined when the serum M-protein level exceeds $3 \mathrm{~g} / \mathrm{dL}$ and/or percentage of plasma cells in the bone marrow ranges from $10 \%$ to $60 \%$. There should be no evidence of associated end-organ damages such as lytic lesions, anemia, renal disease, or hypercalcemia that can be attributed to the underlying plasma cell disorder or other myeloma defining events. Additionally, amyloidosis has to be ruled out. ${ }^{13}$ The patient's anemia was due to iron defi-
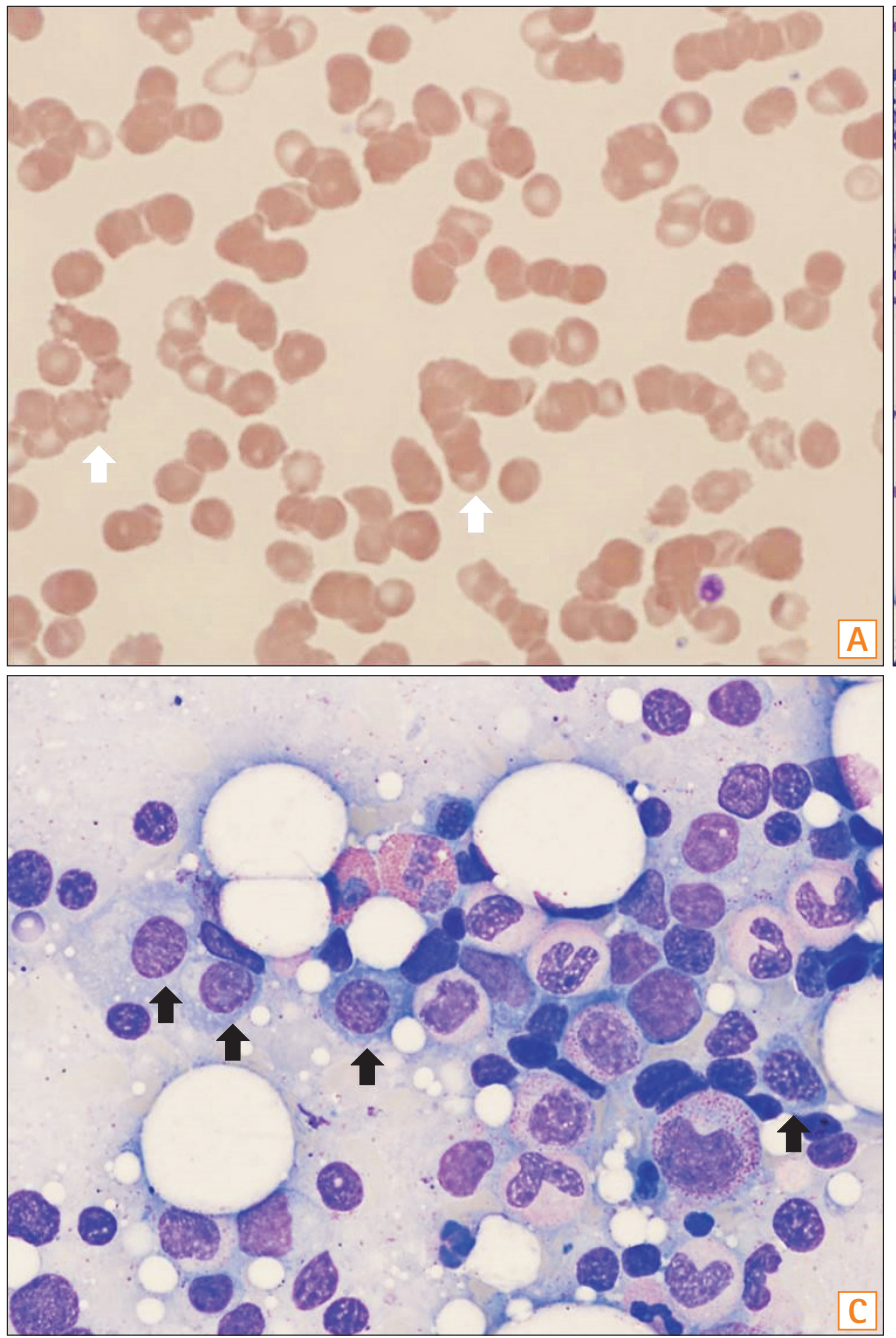

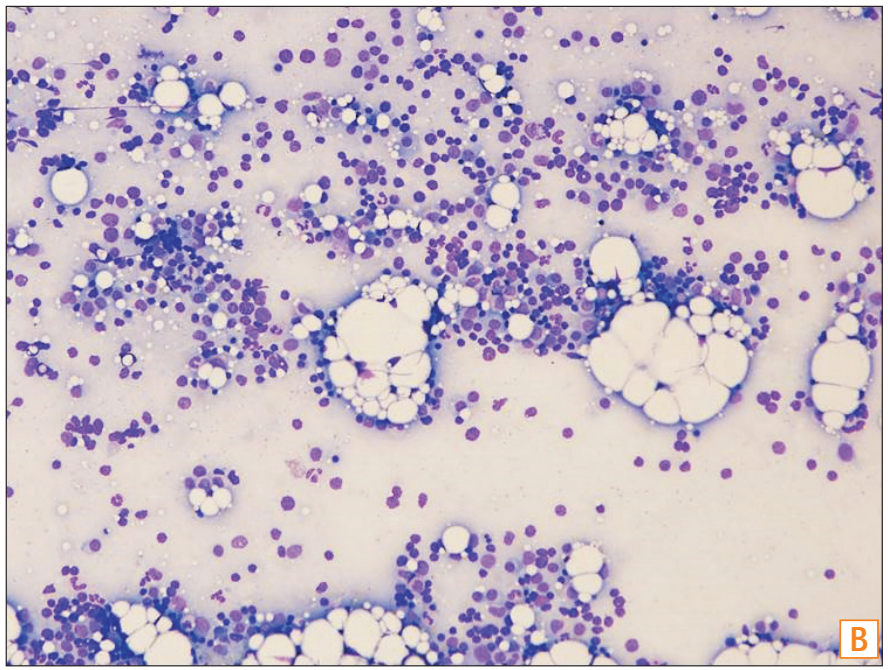

Fig. 2. Peripheral blood smear and bone marrow study. (A) Peripheral blood smear exhibiting marked rouleaux formation (white arrows) (Wright-Giemsa stain, $\times 1,000$ ). (B) Bone marrow aspirate. Plasma cells accounted for $14.0 \%$ of the cell count $\left(H \& E E_{1} \times 200\right)$. (C) Bone marrow aspirate. Plasma cells accounted for $14.0 \%$ of the cell count (black arrows) $(\mathrm{HEE}, \times 1,000)$.

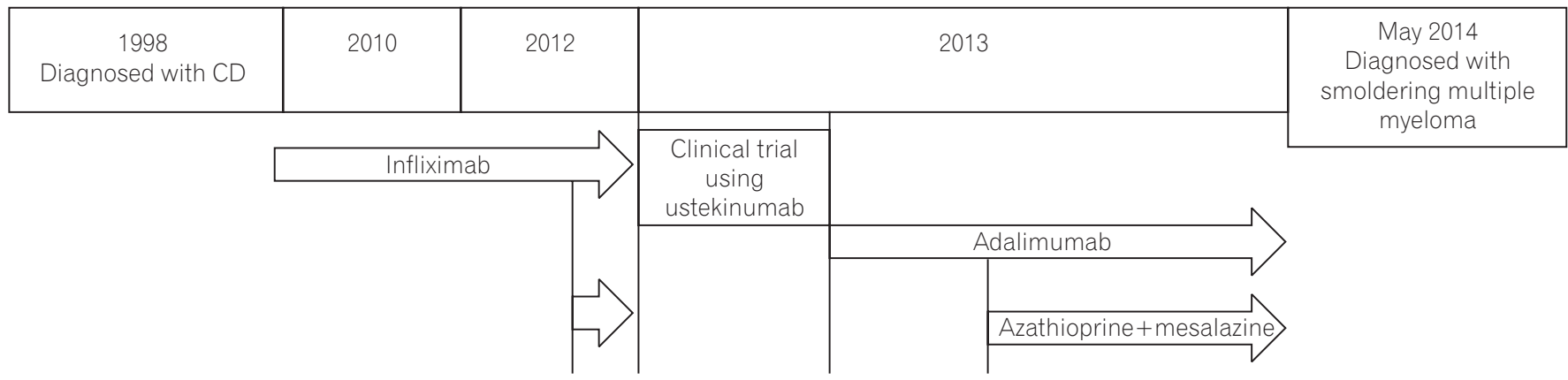

Azathioprine+ mesalazine

Fig. 3. Timeline (in years) showing CD diagnosis, CD treatment, and diagnosis of smoldering multiple myeloma. No records are available for cases before 2010. 
ciency and other end-organ damage was absent. Because the percentage of plasma cells was $14 \%$ on a bone marrow biopsy examination, his disease was classified as SMM. After this diagnosis was established, we discontinued administration of azathioprine.

Adalimumab and mesalazine were reintroduced in June 2014, because patient experienced continuous symptoms of $\mathrm{CD}$. While the patient was on treatment with adalimumab and mesalazine, we tested his complete blood count every 2 months. The complete blood count returned to normal values after 6 months, without any intervention for MM. Because the disease could progress, we performed serum protein electrophoresis, immunofixation, and Ig and free light chain analysis every 3 months. In august 2015, an abnormal zone of restriction was still observed on serum protein electrophoresis and immunofixation. Follow-up M peak, serum IgA level, and serum free $\kappa$ concentration revealed levels of $0.57 \mathrm{~g} / \mathrm{dL}, 9.7 \mathrm{~g} / \mathrm{L}$, and $31.2 \mathrm{mg} / \mathrm{L}$, respectively. Lab values on follow-up examination did not change significantly from the initial values, and end organ damage associated with MM has not been reported so far (Fig. 3).

\section{DISCUSSION}

In IBD, the first line of mucosal defense, including secretory IgA or mucus secretion, is often compromised, resulting in a systemic response to antigens crossing the intestinal mucosal barrier. This leads to an increased ability to activate B-lymphocytes and plasma cells. ${ }^{11,14}$ Such chronic stimulation of B-lymphocytes could explain the causal relationship between MM and IBD. Seven cases with these concurrent conditions have been previously reported in the literature. ${ }^{1,7-12}$ For better characterization of $\mathrm{CD}$ in association with
MM, we conducted a literature review using the PubMed database, using the key words "plasmacytoma," "multiple myeloma," and "Crohn disease." Table 1 summarizes the data of the reported cases. There were five male and three female patients. In terms of CD treatment, only three patients had been receiving immunomodulators. Four cases, including our case, were reported post-1998, when the U.S. Food and Drug Administration approved infliximab for the treatment of $\mathrm{CD}$ in adult patients. MM was diagnosed 45 months to 30 years after the diagnosis of $\mathrm{CD}$, with a median of 14.5 years.

Reynolds et al. ${ }^{1}$ reported a case of MM development related to infliximab use in a patient with CD. Based on the fact that the observed TNF- $\alpha$ serum levels are higher in active patients with MM than in healthy controls, a pilot study on treatment with recombinant human soluble TNF receptor fusion protein (etanercept) in patients with refractory MM was conducted. ${ }^{15,16}$ The results were rather disappointing; the study failed to result in an objective response, and an acceleration of disease was noted in four of the 10 patients. That study suggested that blocking TNF activity could lead to increased proliferation of plasma cells. Additionally, TNF- $\alpha$ has been shown to be a potent inducer of apoptosis in MM cell lines. ${ }^{6}$ Thus, blocking TNF- $\alpha$ activity might be associated with MM development or progression from MGUS to MM. Since there is a possible causal association between TNF- $\alpha$ inhibitors and MM, additional screening parameters such as total serum protein level, serum protein/albumin ratio, urine protein level, urinalysis, and/or serum Ig quantitation might be required before the initiation of TNF- $\alpha$ inhibitor therapy for patients with IBD.

Generally, the average age at which MM occurs is reported as 66 years; however, for our patient, MM occurred at an earlier age of 36 years. ${ }^{17}$ Considering that infliximab and adali-

Table 1. A Summary of Data from Previous Reports on Plasma Cell Disorders in Patients with CD

\begin{tabular}{|c|c|c|c|c|c|c|c|c|}
\hline No. & $\begin{array}{c}\text { Sex/age } \\
\text { (yr) }\end{array}$ & $\begin{array}{l}\text { Interval } \\
\text { from CD }\end{array}$ & Location of CD & CD treatment & Diagnosis & $\begin{array}{c}\text { Monoclonal lg } \\
\text { subtype }\end{array}$ & Treatment & Reference \\
\hline 1 & F/66 & $14 \mathrm{yr}$ & Ileum & Resection & Plasmacytoma & Not specified & NA & {$[7]$} \\
\hline 3 & $M / 36$ & $45 \mathrm{mo}$ & Total colon, ileum & Observation & MM & $\lg G, \lambda$ & NA & [9] \\
\hline 4 & $M / 45$ & NA & Total colon, ileum & Resection & Plasmacytoma & Not specified & NA & [10] \\
\hline 6 & $\mathrm{~F} / 50$ & $5 \mathrm{yr}$ & Small bowel, colon & AZA, 6-MP, IFX & $\mathrm{MM}$ & $\lg G, \kappa$ & VAD, ASCT & [1] \\
\hline 7 & $\mathrm{M} / 50$ & $6 \mathrm{yr}$ & Small bowel & Resection & MM & $\lg G, \lambda$ & VAD, ASCT & {$[12]$} \\
\hline 8 & $M / 36$ & $15 \mathrm{yr}$ & Total colon & AZA, SAS, IFX, ADA & SMM & $\lg A, \kappa$ & Observation & Current study \\
\hline
\end{tabular}

F, female; NA, not available; $M$, male; SAS, sulfasalazine; MM, multiple myeloma; AZA, azathioprine; 6-MP, mercaptopurine; IFX, infliximab; VAD, vincristine adriamycin dexamethasone; $A S C T$, autologous stem cell transplantation; $A D A$, adalimumab. 
mumab were used for the treatment of $\mathrm{CD}$, it is presumed that the occurrence of $\mathrm{MM}$ in our presented case was triggered by TNF- $\alpha$ blockage. However, Mielke et al. ${ }^{18}$ reported that no deterioration of the MM was detected in a patient with pre-existing MM despite treatment with adalimumab for rheumatoid arthritis. They suggested that there is no direct association between treatment with TNF blockers and occurrence of MM. ${ }^{18}$ In our case, adalimumab was continuously used to treat CD despite the presence of SMM because the patient's symptoms such as diarrhea and weight loss were further aggravated. The severity of MM did not change during adalimumab therapy. Another case presented progression from underlying MGUS to myeloma, following infliximab treatment for pyoderma gangrenosum. ${ }^{19}$ Therefore, further studies on the relationship between TNF- $\alpha$ blockage and MM progression are required. In addition, further studies are needed to analyze the difference between various TNF- $\alpha$ blockers and their effects on MM progression. Moreover, when SMM is diagnosed during TNF- $\alpha$ blocker therapy, regular close monitoring of complete blood count, blood chemistry, urinalysis, serum Ig level, serum free light chain level, and serum/urine protein electrophoresis with immunofixation is required.

While all previously reported cases were of IgG subtype $\mathrm{MM}$, this case is different in that the MM was of IgA subtype. However, there is no study reporting the meaning of MM Ig subtype in CD patients. Shareef et al. ${ }^{19}$ reported a case of progression from underlying IgA subtype MGUS to myeloma, following infliximab treatment for pyoderma gangrenosum, and not for CD. However, this case was different from our case, as pre-existing IgA gammopathy progression to myeloma rather than IgA subtype myeloma was caused by infliximab.

In conclusion, $\mathrm{CD}$ and $\mathrm{MM}$, two relatively uncommon diseases, seem to be pathologically related. A better-designed prospective epidemiological study on hematologic malignancy development in patients with CD should be conducted for a better understanding about association between pathophysiology, treatment response, prognosis, and treatment received by these patients. Given the paucity of data, the decision to administer TNF- $\alpha$ blockers to patients with hematologic abnormalities and/or history of other malignancies should be carefully considered.

\section{REFERENCES}

1. Reynolds GJ, Annis KA, de Villiers WJ. Review article: multiple myeloma and inflammatory bowel disease. Dig Dis Sci 2007;52:2022-2028.

2. Carter A, Tatarsky I. The physiopathological significance of benign monoclonal gammopathy: a study of 64 cases. Br J Haematol 1980;46:565-574.

3. Greipp PR, Lust JA. Pathogenetic relation between monoclonal gammopathies of undetermined significance and multiple myeloma. Stem Cells 1995;13 Suppl 2:10-21.

4. Bouhnik Y, Lémann M, Mary JY, et al. Long-term follow-up of patients with Crohn's disease treated with azathioprine or 6-mercaptopurine. Lancet 1996;347:215-219.

5. Farrell RJ, Ang Y, Kileen P, et al. Increased incidence of nonHodgkin's lymphoma in inflammatory bowel disease patients on immunosuppressive therapy but overall risk is low. Gut 2000;47:514-519.

6. Gazitt Y, Shaughnessy P, Montgomery W. Apoptosis-induced by TRAIL and TNF-alpha in human multiple myeloma cells is not blocked by BCL-2. Cytokine 1999;11:1010-1019.

7. Robertson EJ, Al-Kaisi NK, Vareska GJ, Ponsky JL. Plasmacytoma of the ileum complicating Crohn's disease: report of a case and review of the literature. Surgery 1986;100:916-923.

8. Freeman HJ. Tabulation of myeloid, lymphoid and intestinal malignancies in Crohn's disease. Can J Gastroenterol 2002;16:779-784.

9. Nakajima H, Munakata A, Yoshida Y. Extraintestinal cancers in Crohn's disease. Digestion 1990;47:1-7.

10. Ligato S, El-Naggar A, Cleary KR, Manning J. Extramedullary plasmacytoma mimicking primary colonic carcinoma in a patient with Crohn's disease: case report and literature review. Arch Pathol Lab Med 1996;120:279-282.

11. Minami A, Iwai A, Watanabe Y, et al. Two cases of inflammatory bowel disease with multiple myeloma. J Gastroenterol 1999;34:629-633.

12. Liu H, Moreb JS. Association of multiple myeloma and inflammatory bowel diseases. Dig Dis Sci 2008;53:863-864.

13. Rajkumar SV, Dimopoulos MA, Palumbo A, et al. International Myeloma Working Group updated criteria for the diagnosis of multiple myeloma. Lancet Oncol 2014;15:e538-e548.

14. Brandtzaeg P, Halstensen TS, Kett K, et al. Immunobiology and immunopathology of human gut mucosa: humoral immunity and intraepithelial lymphocytes. Gastroenterology 1989;97:1562-1584. 
15. Filella X, Blade J, Guillermo AL, Molina R, Rozman C, Ballesta AM. Cytokines (IL-6, TNF-alpha, IL-1alpha) and soluble interleukin-2 receptor as serum tumor markers in multiple myeloma. Cancer Detect Prev 1996;20:52-56.

16. Tsimberidou AM, Waddelow T, Kantarjian HM, Albitar M, Giles FJ. Pilot study of recombinant human soluble tumor necrosis factor (TNF) receptor (p75) fusion protein (TNFR:Fc; Enbrel) in patients with refractory multiple myeloma: increase in plasma TNF alpha levels during treatment. Leuk Res 2003;27:375-380.
17. Bladé J, Kyle RA. Multiple myeloma in young patients: clinical presentation and treatment approach. Leuk Lymphoma 1998;30:493-501.

18. Mielke F, Schweigert M. Safe adalimumab therapy for rheumatoid arthritis in a patient with pre-existing multiple myeloma. Nat Clin Pract Rheumatol 2008;4:218-221.

19. Shareef MS, Munro LR, Owen RG, Highet AS. Progression of IgA gammopathy to myeloma following infliximab treatment for pyoderma gangrenosum. Clin Exp Dermatol 2012;37:146-148. 